\title{
Trehalose Liposomes Suppress the Growth of Tumors on Human Lung Carcinoma-bearing Mice by Induction of Apoptosis In Vivo
}

\author{
HIDEAKI ICHIHARA, KEIJI KUWABARA and YOKO MATSUMOTO
}

\author{
Division of Applied Life Science, Graduate School of Engineering, Sojo University, Kumamoto, Japan
}

\begin{abstract}
Background/Aim: Previous evidence demonstrates that trehalose liposomes (DMTreC14) composed of $L-\alpha$ dimyristoylphosphatidylcholine (DMPC) and $\alpha$-D-glycopyranosyl- $\alpha$-D-glucopyranoside monomyristate (TreC14) inhibit proliferation and invasion on lung carcinoma (A549 cells) in vitro. Here, we aimed to investigate suppressive effects of DMTreC14 on the growth of tumor on human lung carcinoma bearing mice. Materials and Methods: DMTreC14 composed of 30 mol\% DMPC and 70 mol\% TreC14 were prepared by the sonication method. Anti-tumor activities of DMTreC14 using the subcutaneous and orthotopic graft-bearing mice of A549 cells were investigated in vivo. Results: The remarkable reduction of volume and weight in subcutaneous tumors on subcutaneous lung carcinoma-bearing mice topically administrated with DMTreC14 were obtained. Apoptotic-positive cells in the subcutaneous tumor slice of subcutaneous lung carcinomabearing mice topically administrated with DMTreC14 were observed using TUNEL staining. Lung weights on the orthotopic graft-bearing mice of lung carcinoma intravenously administrated with DMTreC14 were markedly decreased compared to those of the control group. Remarkable decrease in dimensions of tumor area of lung on the orthotopic graft-bearing mice of lung carcinoma intravenously administrated with DMTreC14 was obtained in histological analysis using the hematoxylin and eosin staining. Conclusion: Remarkably high anti-tumor activities of DMTreC14 for the subcutaneous and orthotopic graft-bearing mice of lung carcinoma accompanied with apoptosis were revealed for the first time in vivo.
\end{abstract}

This article is freely accessible online.

Correspondence to: Prof. Yoko Matsumoto, Division of Applied Life Science, Graduate School of Engineering, Sojo University, 4-22-1, Ikeda, Nishi-ku, Kumamoto, 860-0082, Japan. Tel: +81 963263965, Fax: +81 963261331, e-mail: matumoto@life.sojo-u.ac.jp

Key Words: Trehalose liposomes, human lung carcinoma, chemotherapy, in vivo, apoptosis.
Lung cancer is one of the cancers that have high mortality all over the world. Lung cancer is classified into small cell lung carcinoma and non-small cell lung carcinoma. Eighty to ninenty percent of lung cancers are non-small cell lung carcinomas. The efficacy of chemotherapy and the radiotherapy for non-small cell lung carcinoma tends to be low, and surgical resection of cancer is mainly applied for patients in early stage non-small cell lung carcinoma (1-3). Combination chemotherapy is carried out in addition to the surgical resection of cancer for an advanced non-small cell lung carcinoma (1, 3-4). The main anti-cancer drug in chemotherapy for non-small cell lung carcinoma is cisplatin $(1,3)$. Cisplatin-combination chemotherapy use irinotecan, paclitaxel, gemcitabine and vinorelbine $(1,3)$. Because combination chemotherapy may be accompanied with severe side-effects $(1,3)$, anticancer drugs without side effects for the patients with lung carcinoma are desired.

Sugars recognize molecules through a receptor on the cell membrane and play an important role in communication between cell and cell adhesion. Trehalose of disaccharides is included in insects, shrimps, plants and mushrooms. Trehalose has a strong hydration power. A strong binding of trehalose for the water molecule in model of cell membrane has been reported (5). Protection effects for hemolysis by cryopreservation of human red blood cells have been reported (6). A perioperative organ preservation solution using trehalose has been reported (7).

Trehalose liposomes (DMTreC14) have been produced by using sonication after mixing L- $\alpha$-dimyristoylphosphatidylcholine (DMPC) and $\alpha$-D-glycopyranosyl- $\alpha-D-$ glucopyranoside monomyristate (TreC14) in buffer solutions without contamination of an organic solvent (8). Trehalose liposomes inhibited the growth of colon and gastric cancer, hepatic carcinoma and leukemia (8-10). Inhibitory effects and anti-invasive activities of trehalose liposomes against lung carcinoma have been obtained in vitro (11). However, anti-tumor activities of trehalose liposomes on model mice of lung carcinoma in vivo have not yet been elucidated. 
In this study, we investigated the anti-tumor activities of DMTreC14 composed of DMPC and TreC14 using the subcutaneous and orthotopic graft bearing mice of lung carcinoma (A549 cells) in vivo.

\section{Materials and Methods}

Preparation of trehalose liposomes. DMTreC14 were prepared using sonicator (WT-200-M; Honda Electronics, Aichi, Japan) after mixing 30\% mol \% DMPC (purity $>99 \%$; NOF Co. Ltd., Tokyo, Japan) and $70 \%$ mol \% TreC14 (Dojindo Ltd., Kumamoto, Japan) at $45^{\circ} \mathrm{C}$ in $5 \%$ glucose solution. Sample solutions were then sterilized using a $0.45 \mu \mathrm{m}$ cellulose acetate filter (Advantec, Tokyo, Japan). Sample solutions were stored at room temperature $\left(25^{\circ} \mathrm{C}\right)$.

Dynamic light scattering method. The diameter of DMTreC14 was measured with a light scattering spectrophotometer (ELSZ-0, Otsuka Electronics, Japan). Using a He-Ne laser as a light source, a $633 \mathrm{~nm}$ laser line by $10 \mathrm{~mW}$ power was applied with a scattering angle of $90^{\circ}$. The hydrodynamic diameter $\left(d_{\text {hy }}\right)$ of DMTreC14 was calculated by Stokes-Einstein equation $\left(d_{\mathrm{hy}}=\mathrm{kT} /(3 \pi \eta D)\right.$, where $D$ is the diffusion coefficient, $k$ is Boltzmann's constant, $T$ is the absolute temperature and $\eta$ is the viscosity of solvent.

Cell culture. Human non-small cell lung carcinoma (A549) cell lines were obtained from the Riken Cell Bank (Ibaraki, Japan). A549 cells were cultured in D-MEM medium (Life Technologies, Carlsbad, CA, USA) complemented with $10 \%$ fetal bovine serum (FBS, HyClone Laboratories Inc., UT, USA), streptomycin $(50 \mu \mathrm{g} / \mathrm{ml})$ and penicillin $(100 \mathrm{unit} / \mathrm{ml})$. Cells were allowed to grow in an incubator humidified at $37^{\circ} \mathrm{C}$ and at atmosphere of $5 \% \mathrm{CO}_{2}$.

Assessment of anti-tumor activities of DMTreC14 for subcutaneous lung carcinoma bearing mice in vivo. Animals were handled in accordance with the guidelines for animal experimentation of the Japanese law. All animal experiments were approved by the Committee on the Ethics of Animal Experiments of the Sojo University. Balb/c Rag-2/Jak3 double-deficient (Balb/c-R/J) mice were provided by Dr. Seiji Okada (Center for AIDS Research, Kumamoto University, Japan) (12). A549 cells $\left(5.0 \times 10^{6}\right.$ cells $)$ suspended into matrigel (BD Co., USA) were subcutaneously inoculated to dorsal flank of $\mathrm{Balb} / \mathrm{c}-\mathrm{R} / \mathrm{J}$ mice. Animals were randomly separated $(n=4)$ to each group. DMTreC14 were topically administered once a day for 25 days from day 2 . The tumor volume was calculated from (a) smallest diameter and (b) longest diameter following equation:

Tumor volume $(\mathrm{V})=0.5 \times \mathrm{a}^{2} \times \mathrm{b}(13)$.

Subcutaneous tumors were removed from anaesthetized mice after the administration with DMTreC14 and weighed.

TUNEL method. Detection of apoptotic cells was performed on the basis of the TUNEL method using an in situ apoptosis detection kit (ApopTag Plus Peroxidase, Intergen, USA) according to manufacturer's directions. A549 cells $\left(5.0 \times 10^{6}\right.$ cells $)$ suspended into matrigel were subcutaneously inoculated to dorsal flank of Balb/c$\mathrm{R} / \mathrm{J}$ mice. Animals were randomly separated at four to each group. DMTreC14 were topically administered once a day for 25 days from day 2. Subcutaneous tumors were removed from mice under anesthetization after the administration with DMTreC14 and fixed in $10 \%$ formalin solution. The subcutaneous tumors were embedded in paraffin and sectioned at thickness of $5 \mu \mathrm{m}$. The apoptotic cells of subcutaneous tumor were analysed using the TUNEL method and an optical microscope (TS-100, Nicon, Tokyo, Japan).

Assessment of anti-tumor activities of DMTreC14 on the orthotopic graft bearing mice of lung carcinoma in vivo. Balb/c-R/J mice were randomly grouped $(n=8)$ on the basis of body weight on the day of A549 cells inoculation using the stratified randomization method. A549 cells $\left(3.5 \times 10^{5}\right.$ cells $)$ were intravenously inoculated into tail vein to the $\mathrm{Balb} / \mathrm{c}-\mathrm{R} / \mathrm{J}$ mice. DMTreC14 was intravenously administered once a day for 14 days. After 14 days, the lungs were resected from orthotopic graft bearing mice of lung carcinoma under anesthetization and were weighted.

Histochemical analysis. The lungs were resected from orthotopic graft bearing mice of lung carcinoma under anesthetization after 14 days of inoculation of A549 cells, and fixed in 10\% formalin solution. The lungs were embedded in paraffin and sectioned at thickness of $5 \mu \mathrm{m}$. The sections of lungs were stained with hematoxylin and eosin (HE) and observed with a biological microscope (BZ-X700, Keyence, Osaka, Japan). The dimension of tumors was estimated using an image analysis software of BZ-X700 (BZ-H3M, Keyence, Osaka, Japan).

Statistical analysis. Results are presented as mean \pm S.D. Statistical evaluation of data carried out using Student's $t$-test. Values of $p<0.05$ were considered statistically significant.

\section{Results}

Hydrodynamic diameter of DMTreC14. We measured the membrane diameter of DMTreC14 composed of $30 \mathrm{~mol} \%$ DMPC and $70 \mathrm{~mol} \%$ TreC14 on the basis of dynamic light scattering measurements. Distribution of hydrodynamic diameter $\left(d_{\text {hy }}\right)$ of DMTreC14 was about $100 \mathrm{~nm}$ in narrow range. Membrane diameter of DMTreC14 was stable and kept over 4 weeks. Membrane diameter of DMPC was unstable and precipitated after 14 days.

Anti-tumor activities of DMTreC14 for subcutaneous lung carcinoma bearing mice in vivo. We examined anti-tumor activities of topical administration with DMTreC14 for subcutaneous lung carcinoma bearing mice. The subcutaneous tumor volume in mice topically administered with DMTreC14 was calculated. The results are shown in Figure 1. Remarkable reduction ( $p<0.01$ versus control) in subcutaneous tumor volume was obtained in mice topically administered with DMTreC14. There was no significant difference between control and DMPC. Subcutaneous tumors were resected from anaesthetized mice after the administration with DMTreC14 and weighed. The subcutaneous tumor weight of subcutaneous lung carcinoma bearing mice is shown in Figure 2. Subcutaneous tumor weight of mice administrated with DMTreC14 obviously decreased, although that of the untreated control (non-treated) 


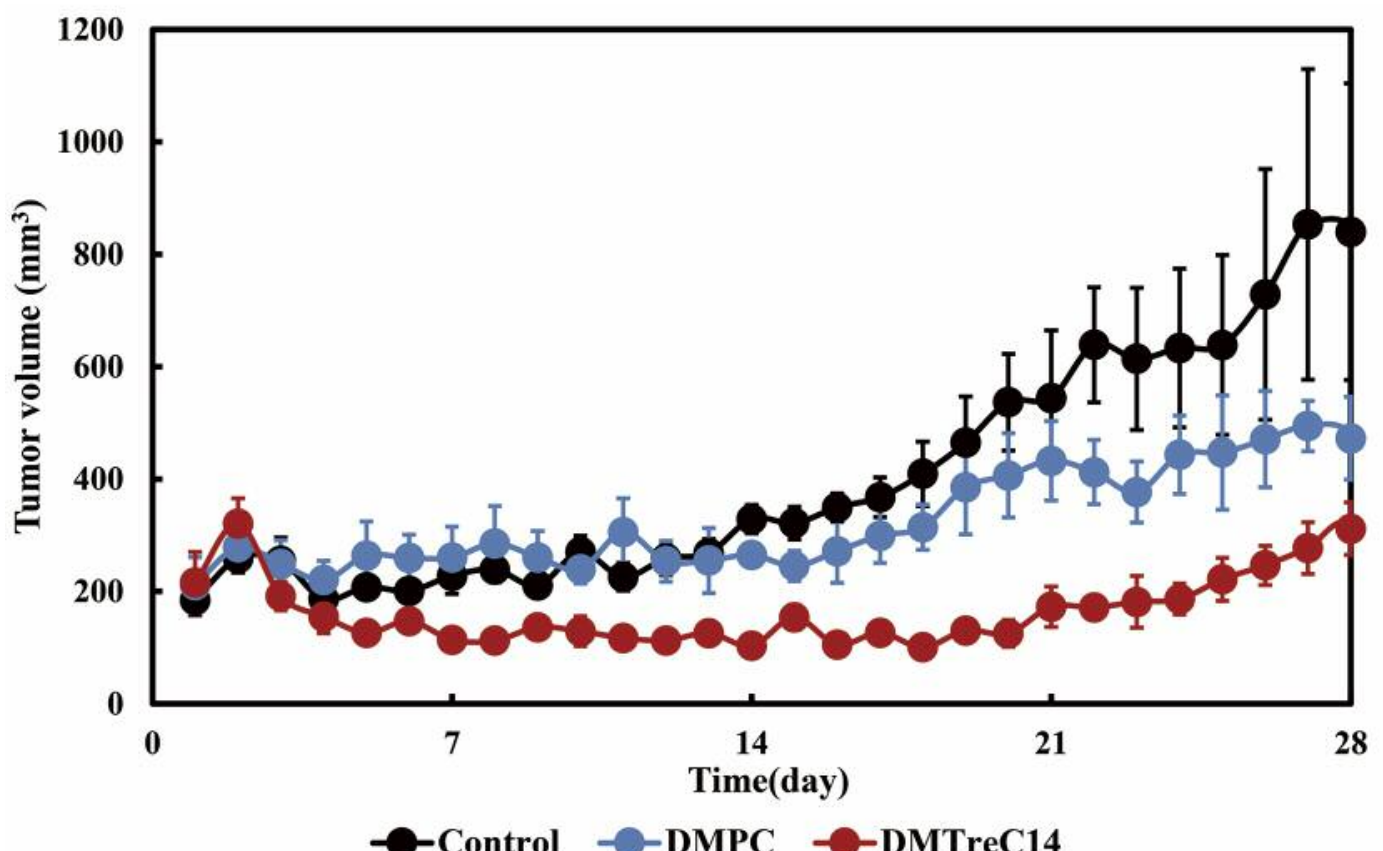

Figure 1. Subcutaneous tumor volume in subcutaneous lung carcinoma bearing mice topically administered with DMTreC14. Dose for DMPC: 3.4 $\mathrm{mg} / \mathrm{kg}$; dose for TreC14: $6.5 \mathrm{mg} / \mathrm{kg}$. Data presented are mean $\pm S . D$.

group increased. There was a significant difference $(p<0.05)$ in the subcutaneous tumor weight between lung carcinomabearing mice administrated with DMTreC14 and the control group. We observed anti-tumor activities of DMTreC14 for subcutaneous lung carcinoma-bearing mice in an autopsy. The results are shown in Figure 3. Whereas the subcutaneous tumor of mice administrated with DMTreC14 remarkably reduced, an increased subcutaneous tumor was confirmed in mice administrated with DMPC and control group.

Induction of apoptosis by DMTreC14 in vivo. We examined the induction of apoptosis by DMTreC14 for subcutaneous tumor in lung carcinoma-bearing mice using the histological analysis of the TUNEL method. The results are shown in Figure 4. A significantly large number of apoptotic cells were observed in the subcutaneous tumor tissues of mice after the administration with DMTreC14, while the apoptotic cells were not observed in the tumor tissues of mice administrated with DMPC and the control group.

Anti-tumor activities of DMTreC14 on the orthotopic graftbearing mice of lung carcinoma in vivo. In order to gain further insight into the antitumor activities of DMTreC14 in vivo, we examined the anti-tumor activities of DMTreC14 using orthotopic graft-bearing mice of lung carcinoma. Lungs were resected from anaesthetized mice intravenously

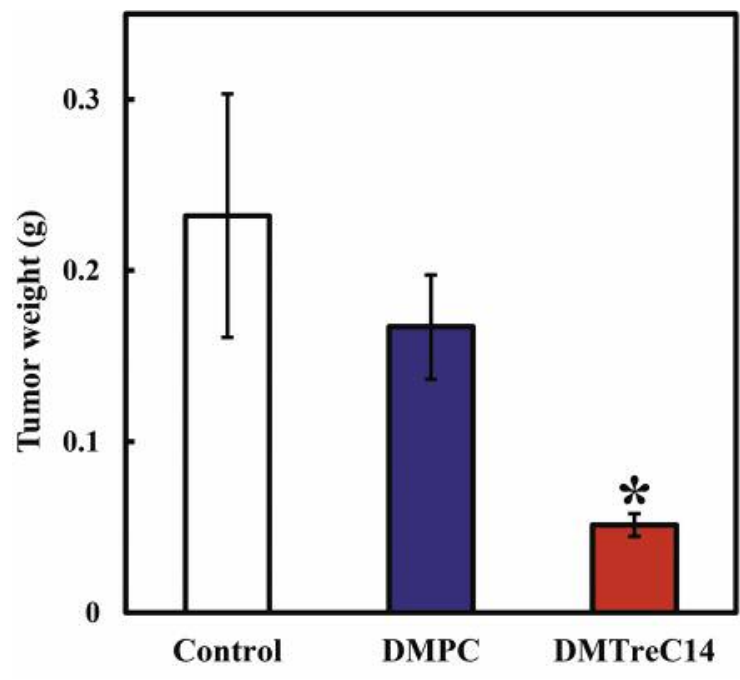

Figure 2. Subcutaneous tumor weight of subcutaneous lung carcinoma bearing mice topically administered with DMTreC14. Dose for DMPC: $3.4 \mathrm{mg} / \mathrm{kg}$; dose for TreC14: $6.5 \mathrm{mg} / \mathrm{kg}$. Data presented are mean $\pm S . D$. $* p<0.05$ (vs. Control).

administered with DMTreC14 and weighed. The relative lung weight of orthotopic-graft bearing mice of lung carcinoma is shown in Figure 5. The relative lung weight in orthotopic graft-bearing mice of lung carcinoma intravenously 


\section{Control}

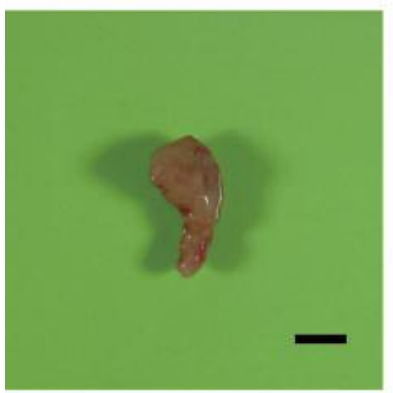

DMPC

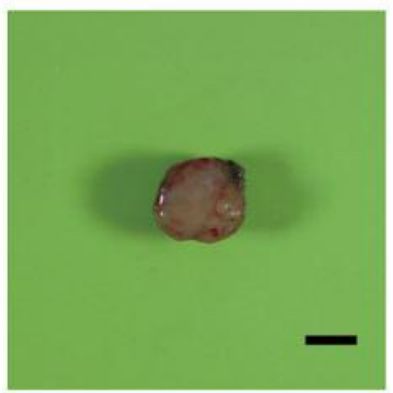

DMTreC14

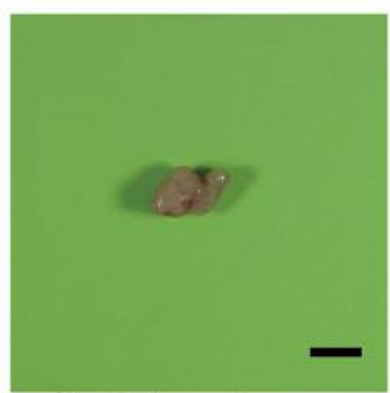

Scale bar : $\mathbf{5} \mathbf{~ m m}$

Figure 3. Photographs of subcutaneous tumor in subcutaneous lung carcinoma-bearing mice topically administered with DMTreC14. Dose for DMPC: $3.4 \mathrm{mg} / \mathrm{kg}$; dose for TreC14: $6.5 \mathrm{mg} / \mathrm{kg}$.

\section{Control}

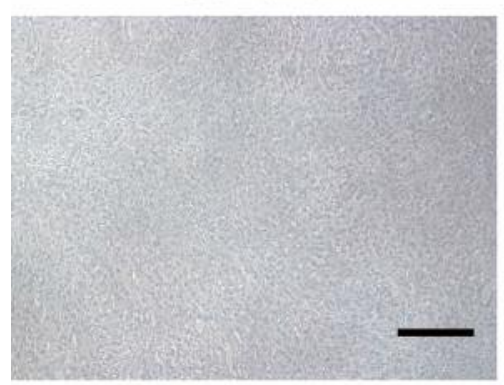

DMPC

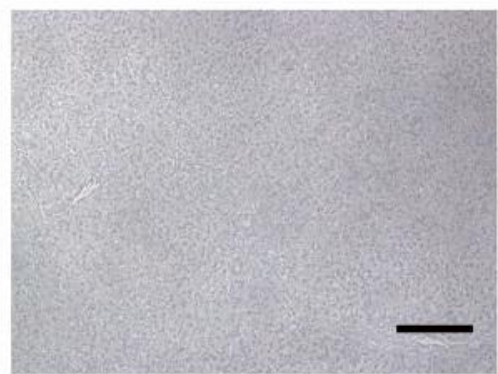

DMTreC14

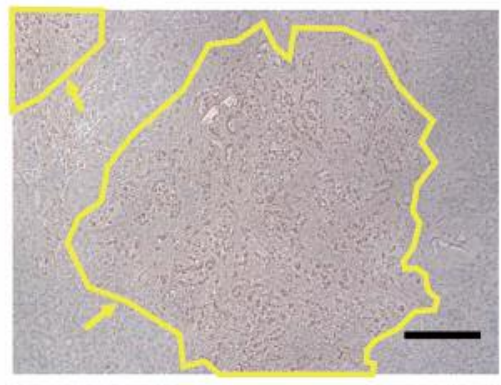

Scale bar : $200 \mu \mathrm{m}$

Figure 4. Micrographs of subcutaneous tumor section in subcutaneous lung carcinoma bearing mice topically administered with DMTreC14 using TUNEL

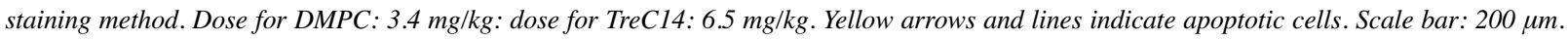

administrated to DMTreC14 was significantly decreased $(p<0.05)$ compared with that of control. There was no significant difference between control and DMPC. We observed anti-tumor activities of DMTreC14 for the bearing mice of lung carcinoma in an autopsy. The results are shown in Figure 6. A decrease of the lung tumorof mice administrated with DMTreC14 was observed, although an enlarged tumor was observed in the lung of mice administrated with DMPC and the control group. The weight loss was not observed in orthotopic graft-bearing mice of lung carcinoma during the duration of administration of DMTreC14, which suggests that DMTreC14 does not cause severe toxicity.

Anti-tumor activities of DMTreC14 on the basis of histological bioanalysis. We evaluated the anti-tumor activities of DMTreC14 for the orthotopic graft-bearing mice of lung carcinoma on the basis of histological bioanalysis in vivo. Lung sections were stained using hematoxylin and eosin

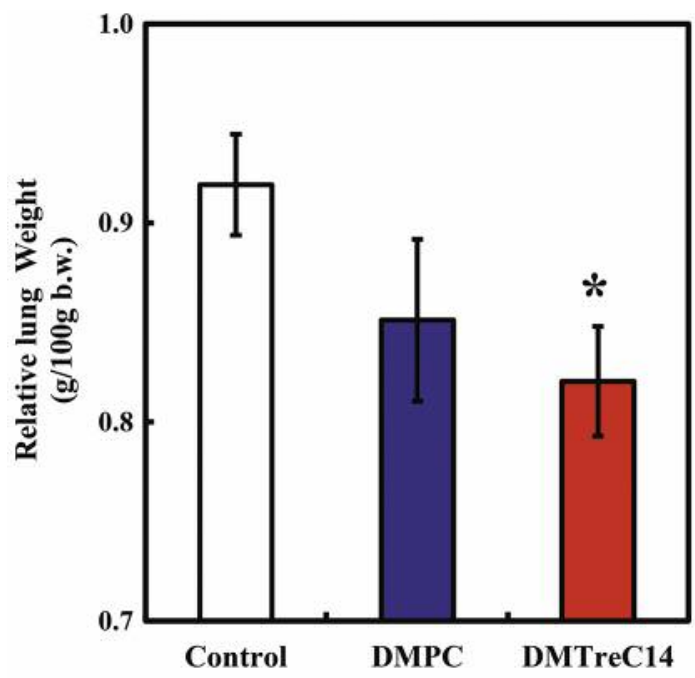

Figure 5. Relative lung weight of orthotopic graft-bearing mice of lung carcinoma intravenously administered with DMTreC14. Dose for DMPC: $13.6 \mathrm{mg} / \mathrm{kg}$; dose for TreC14: $26 \mathrm{mg} / \mathrm{kg}$. Data presented are mean \pm S.D. ${ }^{*} p<0.05$ (vs. Control). 
Control

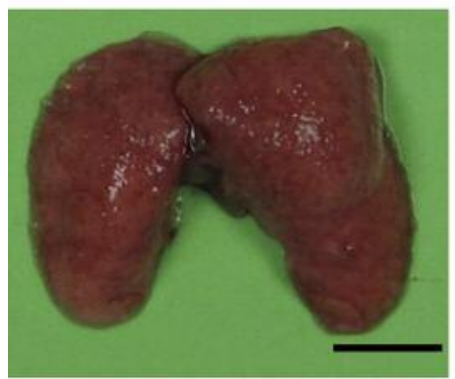

DMPC

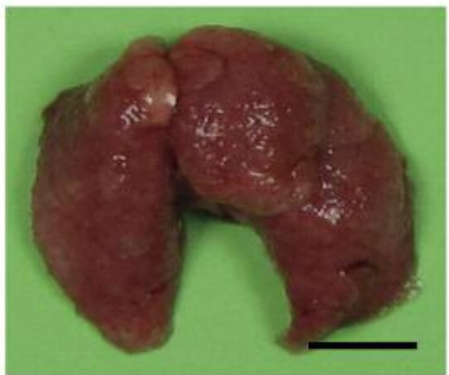

DMTreC14

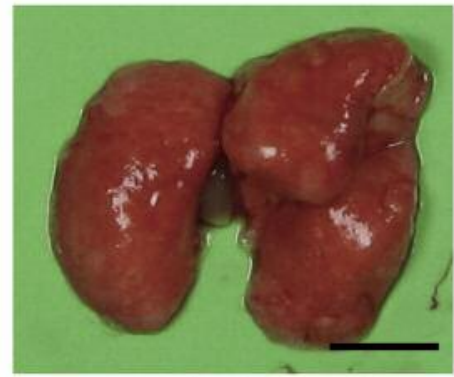

Scale bar : $5 \mathrm{~mm}$

Figure 6. Photographs of lung of orthotopic graft-bearing mice of lung carcinoma intravenously administered with DMTreC14. Dose for DMPC: $13.6 \mathrm{mg} / \mathrm{kg}$; dose for TreC14: $26 \mathrm{mg} / \mathrm{kg}$.
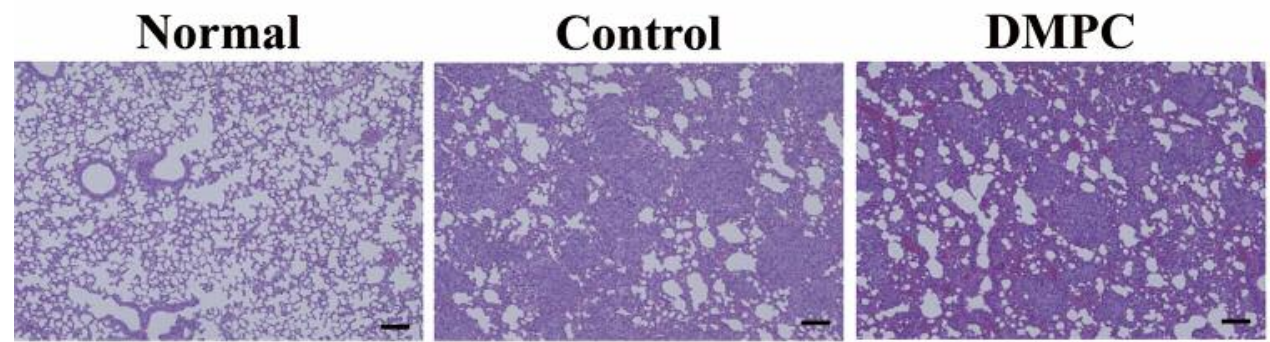

DMTreC14

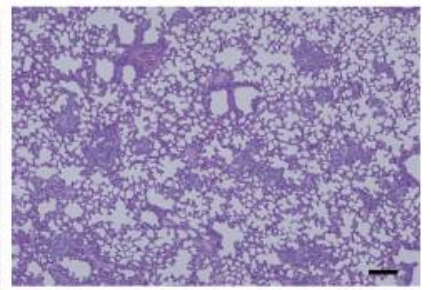

Scale bar : $100 \mu \mathrm{m}$

Figure 7. Micrographs of lung on the orthotopic graft-bearing mice of lung carcinoma intravenously administered with DMTreC14 in histological analysis using the H\&E staining. Dose for DMPC: $13.6 \mathrm{mg} / \mathrm{kg}$; dose for TreC14: $26 \mathrm{mg} / \mathrm{kg}$.

(HE) in order to evaluate the anti-tumor activities of DMTreC14. The results are shown in Figure 7. The significantly large number of tumor cells, were obviously observed in the lung sections of mice administrated with DMPC and the control group. In contrast, a marked reduction of tumor cells was observed in sections of mice administrated with DMTreC14. Lungs of the orthotopic graft-bearing mice of lung carcinoma administrated with DMTreC14 were almost the same as those of normal mice. Furthermore, the dimensions of the tumor area in the lungs of orthotopic graftbearing mice of lung carcinoma were estimated using an image analysis software in biological microscope (BZ-X700). The results are shown in Figure 8. The dimensions of tumor area of the control and DMPC were $1,212 \pm 23 \mathrm{~mm}^{2}$ and $1,202 \pm 40 \mathrm{~mm}^{2}$, respectively, whereas that of DMTreC14 was $916 \pm 41 \mathrm{~mm}^{2}(p<0.05)$. Dimensions of the tumor area of lung of the orthotopic graft bearing mice of lung carcinoma administrated with DMTreC14 was almost the same as that of the normal mice $\left(948 \pm 66 \mathrm{~mm}^{2}\right)$.

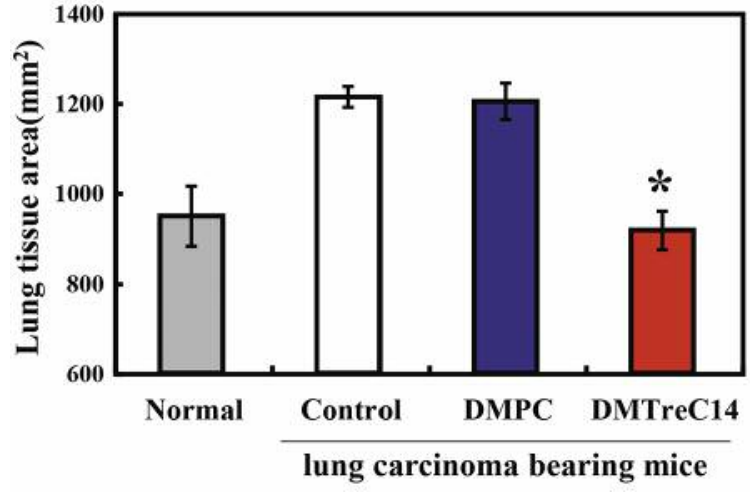

Figure 8. Tissues are of lung on the orthotopic graft-bearing mice of lung carcinoma intravenously administered with DMTreC14. Dose for DMPC: $13.6 \mathrm{mg} / \mathrm{kg}$; dose for TreC14: $26 \mathrm{mg} / \mathrm{kg}$. Data presented are mean \pm S.D. ${ }^{*} p<0.05$ (vs. Control, DMPC). 


\section{Discussion}

Cisplatin-combination chemotherapy is effective for the treatment of patients with lung carcinoma $(1,4)$. The anticancer drug damages normal cells as well as tumor cells and thus causes side-effects. Therefore, anticancer drugs that do not cause side effects in patients are desired.

It is well known that sugars recognize the molecules through a receptor on the cell membrane. The trehalose has a strong hydration power. We examined anti-tumor activities of trehalose liposomes (DMTreC14) composed of DMPC and $\mathrm{TreC} 14$ on the growth of tumors on human lung carcinoma (A549 cells) bearing mice. The remarkable reduction $(p<0.01)$ of volume and weight in subcutaneous tumors of subcutaneous lung carcinoma-bearing mice topically administered with DMTreC14 was observed. Furthermore, the relative lung weight of orthotopic graft bearing mice of lung carcinoma intravenously administrated with DMTreC14 was significantly decreased $(p<0.05)$ as compared with that of the control. Furthermore, to gain further insight into the antitumor activities of DMTreC14 on the lung carcinoma bearing mice, we examined the antitumor activities of DMTreC14 on the orthotopic graftbearing mice of lung carcinoma. We evaluated the anti-tumor activities of DMTreC14 using the orthotopic graft-bearing mice of lung carcinoma on the basis of histological bioanalysis in vivo. Dimensions of tumors of lung tissue sections on the orthotopic graft bearing mice of lung carcinoma that were intravenously administered with DMTreC14 remarkably decreased as shown by histological analysis using the HE staining method. Dimension of lung of the orthotopic graft bearing mice of lung carcinoma administrated with DMTreC14 was almost the same as that of the normal mice. Due to the trehalose having a strong hydration power, specific interaction of the trehalose toward the water molecule on the phospholipid membrane using a model of cell membrane has been reported (5). The movements of water molecules on the cell membrane of cancer cells were more active than normal cells (14). Trehalose liposomes (DMTreC14) inhibited the growth of colon and gastric cancer, hepatic carcinoma and leukemia (810). The thickness of the fixed aqueous layer on the membrane of DMTreCn was larger than that of DMPC (8). These results suggest that DMTreCn could strongly bind to cancer cells by the interaction between strong hydration power of trehalose and water molecule of active movement on cancer cell membrane. Therefore, anti-tumor activities of DMTreC14 could be related to hydration of trehalose in A549 cells of human lung carcinoma bearing mice.

The weight loss was not observed in orthotopic graftbearing mice of lung carcinoma during the duration of administration of DMTreC14. Enhanced accumulation of DMTreCn including fluorescence probe on cell membranes of hepatoma, colonic carcinoma, lymphoma and stomach cancer has been reported (8-10). In contrast, no accumulation of DMTreCn in normal cells was observed. The liposomal medicine has a potential risk of hemolysis of red blood cells. We performed a hemolysis test of DMTreC14 using the red blood cells of normal rats. There was no hemolysis in red blood cells after treatment with DMTreC14. In contrast, hemolysis was observed in the red blood cells treated with DMPC liposomes. We also performed a chronic toxicity test of DMTreC14 using normal healthy mice after the intravenous injection of DMTreC14 for 14 days in vivo. Abnormal findings in weight, blood cell count and biochemical parameters of mice treated intravenously with DMTreC14 were not observed. These results suggest that DMTreC14 may not cause severe side-effects in vivo.

It is well known that apoptosis plays an important role for normal development and for maintenance of homeostasis in tissues. Consequently, cancer cell death by induction of apoptosis is an important potential target for cancer chemotherapy. DMTreC14 induced apoptosis in colon carcinoma, gastric cancer, hepatic carcinoma and leukemia cells (8-10). Furthermore, enhanced activities of caspase-3, -8 and -9 in tumor cells treated with DMTreC14 were obtained in vitro. The decreased mitochondrial transmembrane potential of tumor cells was observed after the treatment with DMTreC14. That is to say, DMTreC14 induced apoptosis by two pathways after fusion of DMTreC14 into the tumor cell membrane. In the first pathway; the apoptotic signal passed through the mitochondria, then caspase- 9 and caspase- 3 , and then reached the nucleus. In the other pathway; the apoptotic signal passed through FAS, then caspase-8 and caspase-3, and then reached the nucleus. We also examined the induction of apoptosis by DMTreC14 of the subcutaneous tumor in lung carcinoma bearing mice after the administration with DMTreC14 using the TUNEL method. A significantly large number of apoptotic cells were observed in the subcutaneous tumor tissues of mice after the administration with DMTreC14. These results suggest that DMTreC14 induced apoptosis of the subcutaneous tumor cells in lung carcinoma bearing mice goes via activation of caspases-3, -8 and -9 and mitochondria.

The results of this study may contribute to the development of chemotherapeutic agents for patients with lung carcinoma in future clinical applications.

\section{Conclusion}

We clearly demonstrated that anti-tumor activities of DMTreC14 accompanied with apoptosis in lung carcinomabearing mice. We showeda remarkable reduction of volume and weight in subcutaneous tumors on subcutaneous lung carcinoma bearing mice topically administrated with 
DMTreC14. Also, we observed aninduction of apoptosis in the subcutaneous tumor slice of subcutaneous lung carcinoma bearing mice administrated with DMTreC14 using TUNEL staining. A marked decrease of lung weights on the orthotopic graft bearing mice of lung carcinoma intravenously administrated with DMTreC14 was also obtained. A remarkable decrease in the dimension of tumors of lung on the orthotopic graft bearing mice of lung carcinoma intravenously administrated with DMTreC 14 was obtained in histological analysis using HE staining method. It is noteworthy that remarkably high anti-tumor activities of DMTreC14 for the subcutaneous and orthotopic graft bearing mice of lung carcinoma accompanied with apoptosis were indicated in vivo.

\section{Conflicts of Interest}

None declared.

\section{Acknowledgements}

The Authors would like to thank Yu Hatae for his technical assistance.

\section{References}

1 Cufer T, Ovcaricek T and O'Brien ME: Systemic therapy of advanced non-small cell lung cancer: major-developments of the last 5-years. Eur. J. Cancer 49: 1216-1225, 2013.

2 Salama JK and Vokes EE: New Radiotherapy and Chemoradiotherapy Approaches for Non-Small-Cell Lung Cancer. J Clin Oncol 31: 1029-1038, 2013.

3 Qin N, Yang X, Zhang Q, Li X, Zhang H, Lv J, Wu Y, Wang J and Zhang S: Efficacy of Icotinib treatment in patients with stage IIIb/IV non-small cell lung cancer. Thorac Cancer 5: 243249,2014

4 Glotzer OS, Fabian T, Chandra A and Bakhos CT: Non-small cell lung cancer therapy: safety and efficacy in the elderly. Drug Healthc. Patient Saf 5: 113-121, 2013.
5 Luzardo MC, Amalfa F, Nuñez AM, Díaz S, Biondi De Lopez $\mathrm{AC}$ and Disalvo EA: Effect of trehalose and sucrose on the hydration and dipole potential of lipid bilayers. Biophys $\mathbf{J} 78$ : 2452-2458, 2000.

6 Stoll C, Holovati JL, Acker JP and Wolkers WF: Synergistic effects of liposomes, trehalose, and hydroxyethyl starch for cryopreservation of human erythrocytes. Biotechnol Prog 28: 364-371, 2012.

7 Chen F, Nakamura T and Wada H: Development of new organ preservation solutions in Kyoto University. Yonsei Med J 45: 1107-1114, 2004.

8 Matsumoto Y, Cao E and Ueoka R: Novel liposomes composed of dimyristoylphosphatidylcholine and trehalose surfactants inhibit the growth of tumor cells along with apoptosis. Biol Pharm Bull 33: 4727-4740, 2013.

9 Matsumoto Y, Cao E and Ueoka R: Growth inhibition by novel liposomes including trehalose surfactant against hepatocarcinoma cells along with apoptosis. Anticancer Res 33: 47274740, 2013.

10 Matsumoto Y, Kuwabara K, Ichihara $\mathrm{H}$ and Kuwano M: Therapeutic effects of trehalose liposomes against lymphoblastic leukemia leading to apoptosis in vitro and in vivo. Bioorg Med Chem Lett 26: 301-305, 2016.

11 Kuwabara K, Ichihara $\mathrm{H}$ and Matsumoto Y: Inhibitory effects and anti-invasive activities of trehalose liposomes on the proliferation of lung carcinoma cells. J Carcinog Mutagen 8: 1000283, 2017

12 Ono A, Hattori S, Kariya R, Iwanaga S, Taura M, Harada H, Suzu S and Okada S: Comparative study of human hematopoietic cell engraftment into BALB/c and C57BL/6 strain of rag-2/jak3 double-deficient mice. J Biomed Biotechnol 2011: 539748, 2011.

13 Shimoda S, Ichihara $\mathrm{H}$, Matsumoto $\mathrm{Y}$ and Ueoka $\mathrm{R}$ : Chemotherapy with hybrid liposomes for human breast tumors along with apoptosis in vivo. Int J Pharm 372: 162-168, 2009.

14 Damadian R, Zaner K, Hor D and Dimaio T: Human tumors detected by nuclear magnetic resonance. Physiol Chem Phys 5: 381-402, 1973 .

Received June 23, 2017

Revised July 9, 2017

Accepted July 10, 2017 